\title{
An improved 2D fem model for straight track corrugation using the mass redistribution method
}

\author{
Fangfang Duan $^{1,2}$, Cedric Pozzolini ${ }^{3}$, Aurelien Saulot ${ }^{1}$ And $^{\text {,a }}$ Yves Berthier $^{1}$ \\ 1 Université de Lyon, CNRS, INSA-Lyon, LaMCoS UMR5259, 69621 Villeurbanne, France \\ 2 Hubei Key Laboratory of Advanced Technology of Automotive Parts, Wuhan University of Technology, 430070, P.R. China \\ 3 Université de Lyon, CNRS, INSA-Lyon, ICJ UMR5208, 69621 Villeurbanne, France
}

Received 6 November 2015, Accepted 17 October 2016

\begin{abstract}
In railway networks, rail corrugation remains a major problem which increases due to heavy traffic under urban conditions: various frictions, train dynamics, etc. But it is difficult to identify the real influence of each factor on both the onset and evolution of such defects on the strength of experiments alone. Thus it is very important to use numerical models to analyze corrugation defects. In this paper, we present an improvement of the finite element model to reproduce more realistically both the onset and evolution of straight track corrugation under transient conditions with the passage of multiple wheels. Furthermore, the application of finite element modeling to the rail-wheel contact problem is optimized by taking the mass redistribution method into account.
\end{abstract}

Key words: Corrugation / wheel-rail contact / finite element method / mass redistribution method

\section{Nomenclature}

\begin{tabular}{|ll|}
\hline$F_{N}$ & Normal load force \\
$F_{t}^{G}$ & Global tangential contact force \\
$F_{n}^{G}$ & Global normal contact force \\
$P_{f}^{G}$ & Global frictional power \\
$v^{\text {wheel }}$ & Translating velocity \\
$v_{\text {sliding }}$ & Sliding velocity \\
$f$ & Frequency \\
$\varepsilon^{P}$ & Plastic deformation \\
$\lambda$ & Wave length \\
$\mu$ & Coulomb friction coefficient \\
$\omega^{\text {wheel }}$ & Angular velocity \\
$\Gamma$ & Global sliding ratio \\
$S_{\text {contact }}$ & Contact surface \\
\hline
\end{tabular}

\section{Introduction}

Straight track corrugation is a periodical rail surface defect that occurs when the wheel-rail contact undergoes transient conditions such as braking or acceleration $[1,2]$.

\footnotetext{
${ }^{a}$ Corresponding author:

aurelien.saulot@insa-lyon.fr
}

Such conditions lead to structural vibrations [3,4] combined with partial slip [5]. The consequence is the development of a periodical wear-like pattern developed on the rail surface [6] which results in rolling noise and reduced rail lifetime [7-9]. Grinding of the rail surface is often necessary to avoid these drawbacks.

Previously, tribological investigations of rails affected by straight track corrugation highlighted periodical plastic deformations coupled with Superficial Tribological Transformation $[10,11]$. From the experimental standpoint, it is difficult to identify the various factors leading to such rail defects and quantify the influence of each factor on the onset and development of straight track corrugation. Tribological investigations into real rails affected by such defects rely on numerical modeling. Up to now, many works have investigated the corrugation defect using numerical modeling. Böhmer and Klimpel considered the influence of plastic deformation as a possible saturation mechanism for corrugation growth [6], and introduced the use of a plane strain finite element model in quasi-static mode. Wen et al. [12-17] combined the non-Hertzian three dimensional rolling contact theory with the theory of friction. They performed a series of simulations of the formation and evolution of corrugations on a curved rail to investigate the effect of train speed and track vibration on corrugation [14,17]. The concept of feedback between the development of corrugation and train-track dynamics were also considered in the 

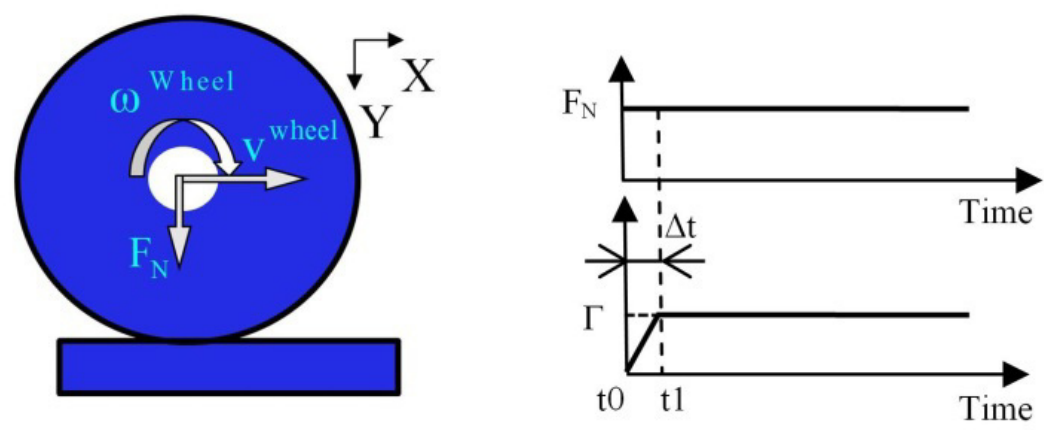

Fig. 1. Evolution of the normal load $F_{N}$ and the global sliding ratio $\Gamma$ imposed in the center of the wheel.

simulation $[13,16,17]$. A 2D dynamic finite element model of wheel-rail contact was also developed to reproduce such periodical defects [19]. The results show specific contact dynamics which caused the onset of periodical plastic deformation on the rail surface as well as a periodical increase of local sliding and temperatures up to several hundred degrees Celsius [8,20,21].

Nevertheless, such simple 2D modeling has been performed with only a single passage of a wheel although in reality several wheels are required to fully develop straight track corrugation. Therefore the previous dynamic finite element model of wheel-rail contact in $2 \mathrm{D}$ will be adapted to allow multiple wheel passages.

Furthermore, classical space-time discretization for this rail-wheel contact problem combines finite element space approximation and time-stepping schemes. In this framework, most methods exhibit spurious oscillations and/or poor behavior in the long term. The modified mass method proposed in References [22-24] is a space semi-discrete formulation that overcomes these two difficulties: the mass term is modified (the mass associated with the normal d.o.f. at the contact boundary is set to zero), which prevents the inertial forces from triggering spurious oscillations, and guarantees the well-posedness of the semi-discrete elastodynamics contact problem with Coulomb friction. Therefore the previous dynamic finite element model of a wheel-rail contact in 2D will be adapted to take into account the modified mass method to increase its accuracy. This paper will present an improvement of the previous work [19] to reproduce both the onset birth and evolution of straight track corrugation under transient conditions more realistically by taking into account both an improved method to solve the rail-wheel contact problem in finite element modeling, and multiple wheel passages

\section{Numerical model}

\subsection{Description the model}

To reproduce the wheel-rail contact problem, the Abaqus toolbox with the "implicit dynamic finite element code Abaqus Standard" (time implicit formulation) is used to build a model of the frictional contact between two 2D-deformable bodies: a rolling disc (a wheel) with elastic behavior and a body (a rail) with elasto-plastic behavior (Fig. 1).

In this paper, iterative schemes for discretizing (in both time and space) versions of systems of the hyperbolic variable inequalities are used to model the frictional elasto-visco-plastic dynamic contact problem. The Hilber-Hughes-Taylor (HHT) implicit scheme is applied for the time discretization and the numerical damping coefficient is set to zero. A classical quadrangular finite element method is used for the space discretization. The inequalities governing the plastic behavior are dealt with by Lagrange multipliers and the radial return method. The inequalities of the dynamic contact problem are solved using Lagrange multipliers: one multiplier for the non-penetration normal condition and one multiplier for the standard Coulomb friction law without penalization or regularization of the tangential stress on the tangential velocity component. Also, a 2D-stress 4node linear plane strain quadrilateral mesh is used in the model. The mesh size is chosen as $0.1 \times 0.1 \mathrm{~mm}$ at the wheel-rail contact area.

Sliding conditions are imposed by increasing the angular velocity $\omega^{\text {wheel }}$ of the center of the disc while the translating velocity $v^{\text {wheel }}$ remains constant. In order to study the contact dynamics (i.e. instability states [19]) occurring under transient conditions, the global stiffness of the train-track system is not considered in this study.

Before the sliding step is applied, the wheel rolls without sliding over the rail at a constant velocity while a constant normal load is applied at the center of the wheel and the model reaches a stable state of pure rolling conditions.

The simulation protocol (Fig. 1) provides the evolution of the global contact conditions: at the beginning of this sliding step $t=t 0$, the wheel rolls without sliding on the rail at a constant linear speed $v^{\text {wheel }}=R^{\text {wheel }} \omega^{\text {wheel }}=$ $17 \mathrm{~m} . \mathrm{s}^{-1}$, then the $\omega^{\text {wheel }}$ imposed at the center of the wheel is increased within a short time $\Delta t[t 0, t 1]$ to create the global sliding conditions between the wheel and the rail. The consequence is an imposed global sliding ratio $\Gamma$ :

$$
\Gamma=\frac{\left|R^{\text {wheel }} \omega^{\text {wheel }}-v^{\text {wheel }}\right|}{v^{\text {wheel }}}
$$


Table 1. Characteristics of the rotating disc (wheel) and the deformable body (rail).

\begin{tabular}{ccc}
\hline & $\begin{array}{c}\text { Deformable rolling } \\
\text { disc (wheel) }\end{array}$ & $\begin{array}{c}\text { Deformable } \\
\text { body (rail) }\end{array}$ \\
\hline Young's modulus $E(\mathrm{GPa})$ & 205 & 205 \\
Poisson's ratio $\Gamma$ & 0.3 & 0.3 \\
Density $\rho\left(\mathrm{kg} \cdot \mathrm{m}^{-3}\right)$ & 7850 & 7850 \\
Dimensions $(\mathrm{mm})$ & $R_{\mathrm{int}}^{\text {wheel }}=70$ & $H_{\text {rail }}=150$ (Height) \\
& $R_{\mathrm{ext}}^{\text {wheel }}=430$ & $L_{\text {rail }}=2340$ (Length) \\
\hline
\end{tabular}

Table 2. The simulation parameters.

\begin{tabular}{cc}
\hline Normal load at the center of the & 8000 \\
wheel $F_{N}(\mathrm{~N})$ & \\
Global sliding ratio $\Gamma$ & $20 \%$ \\
Transient time to apply sliding & $0.1 \times 10^{-3}$ \\
condition $\Delta t(\mathrm{~s})$ & \\
Coulomb friction coefficient $\mu$ & 0.3 \\
\hline
\end{tabular}

The characteristics of both parameters and boundary conditions are shown in Tables 1 and 2 .

\subsection{Introduction of the mass redistribution method (MRM)}

According to the analysis of the numerical results obtained by the previous classical method of approximation, it could be concluded that the corrugation defects do not decrease so quickly in reality. It should be remembered that the effective mechanical energy dissipation in the wheel-rail model is due to friction and plasticity. From the numerical viewpoint, the time integration scheme and mesh approximation should introduce virtual numerical damping. Classical space-time discretization for contact problems combines finite element space approximation and time-step schemes. In Reference [22], the authors studied the numerical instabilities caused by spatial semidiscretization for linear elastodynamic frictionless contact problems in the framework of small deformations. It was shown that the main cause of ill-posedness is due to the inertia of the nodes on the contact boundary. Indeed, it is known that the velocities may be discontinuous when an impact occurs, but the velocities are regularized by a classical finite element discretization of the displacement, with the mass concentrated at the nodes. Then an original method which consists in redistributing the mass near the contact boundary is proposed to recover the wellposedness of the semi-discrete problem and ensure the solution is energy conserving for elastic frictionless contact problems. Moreover, the semi-discrete problem in space reduces to a differential inclusion with a unique Lipschitz continuous solution (rather than to a measure of differential inclusion as in the standard semi-discretization), and this solution can be easily approximated by a numerical time scheme. Finally the redistributed mass matrix conserves the total mass, the center of gravity and the inertia momenta, but is built so that there is no inertia effect for the contact nodes in their normal contact conditions. Furthermore the MRM eliminates spurious oscillations on the contact boundary and conforms to the non-penetration constraint.

Better understanding of the origin of ill-posedness was given by Renard [25] as an adapted modified mass method (based on the MRM, and named "singular dynamic method"). It consists in performing different space semi-discretizations for the velocity and displacement. Then the mass term is modified (in such a way that the mass matrix is singular) and the contact condition is enforced by a variable inequality. This mass modification prevents inertial forces from triggering spurious oscillations at the boundary. Furthermore, the system tends to conserve its energy in the frictionless contact problem, which ensures good long-term behavior. The central theorem proved in Reference [25] for thin structures can be easily extended to $2 \mathrm{D}$ and $3 \mathrm{D}$ impact problems. It confirms that the numerical dissipation is due to spacediscretization and there is no need to introduce an impact law to stabilize the scheme (as was done for rigid body dynamics problems with impact and friction).

The method proposed in this paper is to apply the mass redistribution method introduced in Reference [24] to the rail/wheel model. The mass matrix terms are set to zero only on the node corresponding to the unilateral contact condition, not on the friction one. Furthermore, the mass matrix is kept symmetric and positive semi-definite. Indeed the well-posedness of the fully discrete problem fails when the mass redistribution method is applied to both unilateral contact and friction conditions.

In Reference [22] a simple algorithm was proposed to build the redistributed mass matrix while preserving the main characteristics of the mass matrix (total mass, center of gravity and moments of inertia).

Given that there is no convergence theorem or uniqueness result for the general impact problem, the following convergence trends show that the MRM selects a better conservative numerical solution. Indeed, as the HHT scheme was used for the time discretization and the damping numerical coefficient was set to zero, conformity with the mechanical dissipation was ensured due to friction or plasticity, and the purely numerical loss of energy is corrected.

\subsection{Wheel-rail contact instability states}

Based on a previous study by Saulot et al. [19], the wheel-rail contact model was built to highlight the instability state occurring under sliding conditions using the protocol described in Section 2.1. According to a classical 
rail material law such as R260, a linear elasto-plastic law with isotropic linear hardening properties is used for the rail material: $\sigma_{\text {ep }}=55+250 \varepsilon^{\mathrm{P}}$, with $\sigma_{\text {ep }}$ being the equivalent stress in $\mathrm{MPa}$ and $\varepsilon_{\mathrm{p}}$ the equivalent plastic strain. The convergence criteria are the same as those used in Saulot et al. [19].

In order to highlight the instability states occurring in the contact patch, we obtain the temporal evolution of the global tangential contact force $F_{t}^{G}$, which is the sum of all the local tangential contact forces $F_{t}^{\text {node(i) }}$ at each node of the contact surface:

$$
F_{t}^{G}=\sum_{i} F_{t}^{\text {node(i) }}
$$

To help determine whether or not the instability states lead to surface degradation, the local frictional power $P_{f}^{\text {node(i) }}$ is computed at each node(i) of the contact patch by:

$P_{f}^{\text {node(i) }}=\left|\sigma_{\text {shear }}^{\text {node }(\mathrm{i})} S_{\text {contact }}^{\text {node(i) }}\right| \cdot\left|v_{\text {sliding }}^{\text {node }(\mathrm{i})}\right|=\left|F_{t}^{\text {node(i) }}\right| \cdot \mid v_{\text {sliding }}^{\text {node(i) }}$

with $S_{\text {contact }}^{\text {node(i) }}$ being the elementary contact surface around each node (i) equal to the product of the unitary thickness of the wheel in 2D with the node spacing in the contact patch, $\sigma_{\text {shear }}^{\text {node(i) }}$ is the shear stress at each node(i) and $v_{\text {sliding }}^{\text {node(i) }}$ is the local relative sliding velocity between the wheel and the rail at node(i) in the contact patch. The sum of all $P_{\mathrm{f}}^{\text {node(i) }}$ at each node(i) of the contact is equivalent to the classical global frictional power $P_{\mathrm{f}}^{\mathrm{G}}[18,26]$ given by the relations:

$$
P_{f}^{G}=\left|F_{t}^{G}\right| \cdot\left|v_{\text {sliding }}^{G}\right|=\sum_{i} P_{f}^{\text {node(i) }}
$$

with $\left|v_{\text {sliding }}^{\mathrm{G}}\right|=\Gamma\left|v^{\text {wheel }}\right|$ being the global relative sliding velocity between the rail and wheel.

The slip instability phase is characterized by the evolution of the global tangential contact force $F_{t}^{G}$ presented in Figure 2a. From t0 to t1, when the slip ratio is adapted to the wheel, there is a high frequency of about $5000 \mathrm{~Hz}$ at the beginning of the slip instability states, caused by the instability of instantaneous contact status shifts from stick status to slip status. From $t 0$ to $t 1, F_{t}^{G}$ increases to reach a maximum value of $2400 \mathrm{~N}$ which is a function of both the constant Coulomb friction coefficient $\mu$ (in this case equal to 0.3 ), and the normal force $F_{N}$ of $8000 \mathrm{~N}$. The instability state has a frequency of $527 \mathrm{~Hz}$, which corresponds to the frequency of the 1st torsional mode of the wheel; the shape of this mode is a classical torsional movement of wheel around its center. According to the comparison between the shape of the mode and the behavior of the wheel during this state, we can see that all the nodes in the contact patch are in sliding status, which was called "slip state".

The global frictional power $P_{f}^{G}$ for the slip state is presented in Figure $2 \mathrm{~b}$. The result shows high oscillations with a frequency identical to $F_{t}^{G}$ (see Fig. 2a). The mean
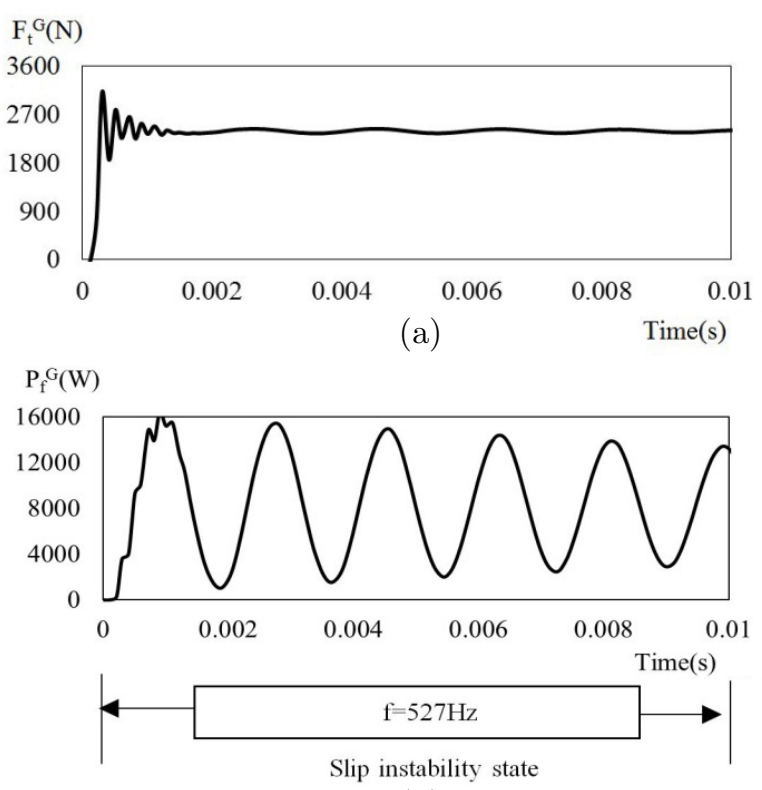

(b)

Fig. 2. Evolution of (a) the global tangential contact force $F_{t}^{G}$ and (b) the global frictional power $P_{f}^{G}$.

value of $P_{f}^{G}=8000 \mathrm{~W}$ is very close to the theoretical global frictional power $P_{f \text { theory }}^{G}=8160 \mathrm{~W}$ which is due to the theoretical maximum tangential contact force, according to both the normal load and friction coefficient, and the sliding velocity imposed at the center of the wheel.

Before the transient conditions occur, the wheel rolls without sliding on the surface of the rail. Figure 3a shows that nearly null plastic deformation occurs. The slip instability state occurs when the transient condition is applied. In the latter case, periodical plastic deformations are highlighted on the rail surface (see Fig. 3b) with a length of $\lambda=30 \mathrm{~mm}$. The maximum plastic deformation is about $0.1 \%$ in slip state. The plastic deformation is produced consecutively to the transient velocity oscillations in the contact path.

The wavelength of the phase corresponds to the frequency of the instability state $f=527 \mathrm{~Hz}$, as a function of the translating velocity $v_{\text {wheel }}=17000 \mathrm{~mm} \cdot \mathrm{s}^{-1}$. Compared with the results obtained under pure rolling condition (Fig. 3a), the periodical plastic deformations in the slip phase (Fig. 3b) show that the transient condition is the reason underlying why the rail undergoes plastic deformation. The results obtained with Abaqus Standard (see Figs. 2 and 3) are very close to those obtained previously by Saulot et al. [19].

These results show that the numerical tool could efficiently represent the periodical plastic deformation matching the corrugation defect. However, in Figure 2, the maximum value of $P_{f}^{G}$ tends to dissipate quickly within $0.01 \mathrm{~s}$ since there is no numerical damping control applied in the simulation. The results obtained in the slip phase show a major problem of quick energy dissipation, which does not conform to the reality of straight track corrugation. According to Akira et al. [27], the 


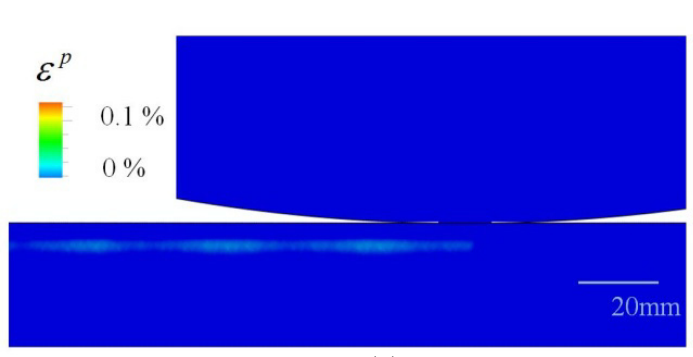

(a)

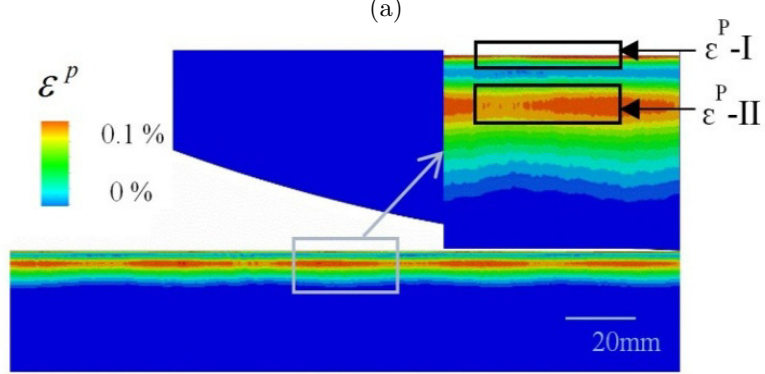

(b)

Fig. 3. Plastic deformation of (a) pure rolling state, (b) slip instability state at $20 \%$ sliding rate with a friction coefficient of 0.3 in the $2 \mathrm{D}$ model.

corrugation defect can be quite constant in the long term, and leads to the deterioration of the profile's geometry with the passage of hundreds and even thousands of wheels. Therefore, in what follows we attempt to find a method to solve the unusual energy dissipation problem in wheel-rail dynamic contact problems. For the sake of clarity, the current results (see Figs. 2 and 3) obtained from the wheel-rail contact model are referred to as the results from the original model (OM). The results obtained from the model that applies the mass redistribution method are referred to as MRM.

\subsection{Convergence of the model with MRM}

Firstly, the same characteristics of both parameters and boundary conditions described in Section 2.1 are applied in the numerical model with MRM.

In the following, the convergence trend of the model with MRM is studied with different time increments and different mesh sizes. The convergence criteria are the same as those used by Saulot et al. [19] and the same as those used for the original model (OM). The latter are detailed in Table 4.

Table 3 shows that the model with MRM needs a shorter time increment $(1 \mathrm{e}-7 \mathrm{~s})$ to converge compared to the original model (OM). This means that the model using MRM costs more CPU time for a full simulation. The case with $\Delta t_{\text {auto }}$ means that the time increment is automatically adjusted by Abaqus Standard from an initial incremental time. The size of the subsequent adjustments made to the time increments depends on how long it takes to complete the simulation.

Secondly, in addition to $\Delta t_{\text {auto }}$ for the size of the subsequent time increments, the trend of convergence with different mesh sizes is studied in the model with MRM.
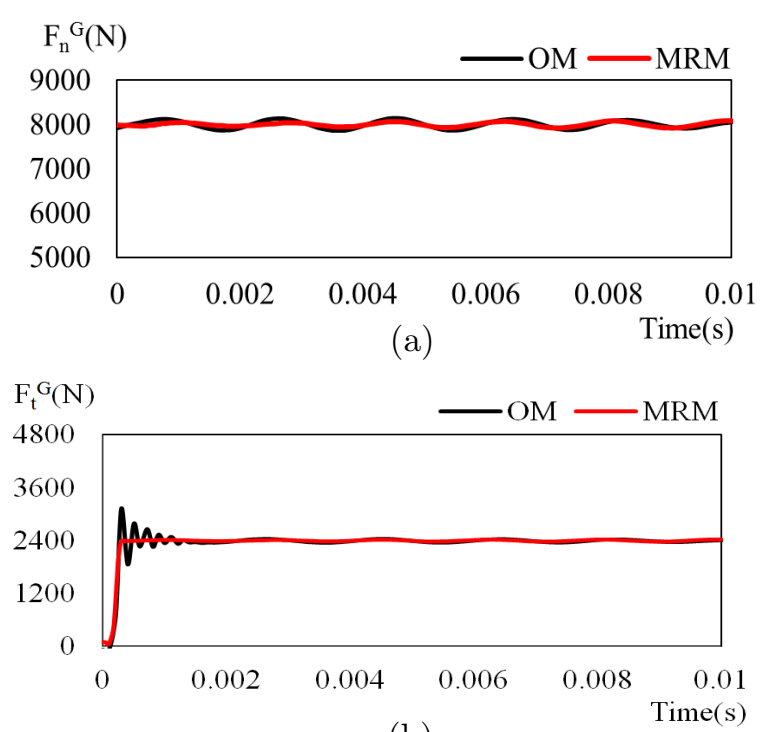

(b)

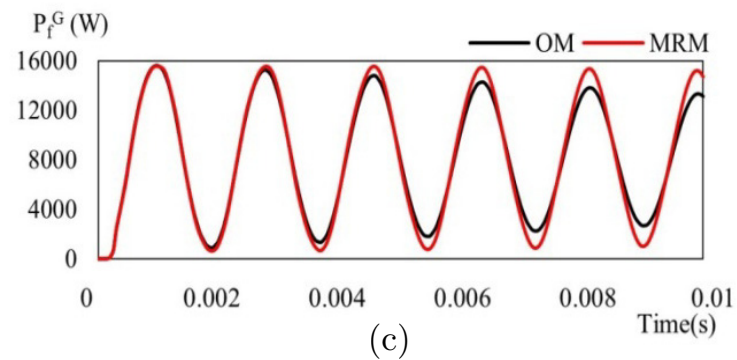

Fig. 4. Comparison of (a) global normal contact force, (b) global tangential contact force, (c) global frictional power between the original model $(\mathrm{OM})$ and the mass redistribution model (MRM).

According to the results computed with different mesh sizes (Tab. 4), all the results with different mesh sizes are compared with a mesh $0.1 \times 0.1 \mathrm{~mm}$. The relative values such as global tangential contact force $\left(F_{t}^{G}\right)$, global normal contact force $\left(F_{n}^{G}\right)$, global frictional power $\left(P_{f}^{G}\right)$, contact pressure $\left(C_{\text {PRESS }}\right)$, and plastic deformation $\left(\varepsilon^{P}\right)$ are shown in Table 4 . There is a great difference between the three mesh sizes in the original model $(\mathrm{OM})$. On the contrary, the results obtained with MRM are influenced only slightly. Therefore, the convergence trend of the model with MRM does not greatly depend on mesh refinement. However, considering that the value of $\varepsilon^{\mathrm{P}}$ is extremely small (about $0.1 \%$ ), the quality of the results depend on a finer mesh size.

Thus the results presented in the following use a mesh size of $0.1 \times 0.1 \mathrm{~mm}$ and automatic time increment to optimize both the computation time and the accuracy of the results obtained.

\subsection{Global results obtained with both $\mathrm{OM}$ and MRM}

The global normal contact force $F_{n}^{G}$ (Fig. 4a) shows that MRM has reduced the inertia effect under normal contact conditions. The curve of $F_{n}^{G}$ in MRM has lower 
Table 3. Different time increments influence on the model convergence tendency.

\begin{tabular}{ccccccccc}
\hline & \multicolumn{2}{c}{$\Delta t_{1}(1 \mathrm{e}-5 \mathrm{~s})$} & $\Delta t_{2}(1 \mathrm{e}-6 \mathrm{~s})$ & \multicolumn{2}{c}{$\Delta t_{3}(1 \mathrm{e}-7 \mathrm{~s})$} & \multicolumn{2}{c}{$\Delta t_{\text {auto }}$} \\
\cline { 2 - 9 } & OM & MRM & OM & MRM & OM & MRM & OM & MRM \\
\hline Mesh $0.1 \times 0.1(\mathrm{~mm})$ & $\times$ & $\times$ & $\sqrt{ }$ & $\times$ & $\sqrt{ }$ & $\sqrt{ }$ & $\sqrt{ }$ & $\sqrt{ }$ \\
Mesh $0.2 \times 0.2(\mathrm{~mm})$ & $\sqrt{ }$ & $\times$ & $\sqrt{ }$ & $\times$ & $\sqrt{ }$ & $\sqrt{ }$ & $\sqrt{ }$ & $\sqrt{ }$ \\
Mesh $0.4 \times 0.4(\mathrm{~mm})$ & $\sqrt{ }$ & $\times$ & $\sqrt{ }$ & $\times$ & $\sqrt{ }$ & $\sqrt{ }$ & $\sqrt{ }$ & $\sqrt{ }$ \\
\hline
\end{tabular}

$\times$ Calculation aborted $\sqrt{ }$ Calculation completed.

Table 4. Comparison of the results with different mesh sizes.

\begin{tabular}{cccccccc}
\hline & Mesh size $(\mathrm{mm})$ & $F_{\mathrm{t}}^{\mathrm{G}}$ & $F_{\mathrm{n}}^{\mathrm{G}}$ & $v_{\text {sliding }}$ & $P_{\mathrm{f}}^{\mathrm{G}}$ & $C_{\text {Press }}$ & $\varepsilon^{\mathrm{P}}$ \\
\hline \multirow{2}{*}{$\mathrm{OM}$} & Mesh $0.2 \times 0.2$-Mesh0.1 $\times 0.1$ & + & + & ++ & ++ & ++ & ++ \\
& Mesh $0.4 \times 0.4-$ Mesh0.1 $\times 0.1$ & + & + & ++ & ++ & +++ & +++ \\
\hline \multirow{2}{*}{ MRM } & Mesh $0.2 \times 0.2$-Mesh $0.1 \times 0.1$ & 0 & 0 & 0 & 0 & ++ & ++ \\
& Mesh $0.4 \times 0.4-$ Mesh0.1 0.1 & 0 & 0 & + & + & +++ & +++ \\
\hline
\end{tabular}

0 Relative value lower than $1 \%$.

+ Relative value lower than $10 \%$.

++ Relative value lower than $20 \%$.

+++ Relative value lower than $50 \%$.

Mesh size impact.

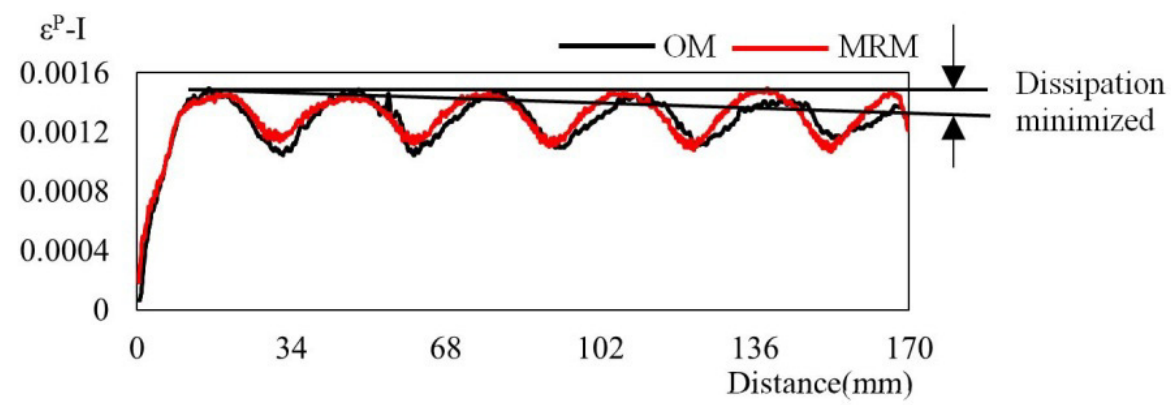

Fig. 5. Comparison of the plastic deformation on the rail surface between the original model (OM) and the mass redistribution model (MRM).

vibration amplitude than the curve in OM (Fig. 4a). The amplitude of the global tangential contact force $F_{t}^{G}$ (see Fig. 4b) in the slip oscillatory state changes little compared with the results obtained in OM and MRM. From the former study, the high frequency at the onset of the sliding state is due to the oscillation of the contact status. The oscillation of the curve $F_{t}^{G}$ (see Fig. 4b) at high frequency was decreased in MRM. This result shows that MRM can dissipate spurious oscillations under contact conditions.

Moreover, the global frictional power $P_{f}^{G}$ computed with both $\mathrm{OM}$ and MRM (Fig. 4c) shows that MRM is less dissipative than OM, as it was expected. In Abaqus, extra and artificial numerical damping is used automatically to ensure that the original model converges. The energy injected in the model through the transient conditions is conserved much better with the application of MRM than with OM. The local contact conditions and consequences are more realistic. Indeed, the equivalent plastic deformation reproduced on the rail surface (Fig. 5) is much more constant through time with MRM. The plastic deformation goes from $0.15 \%$ to $0.13 \%$ over the
$150 \mathrm{~mm}$ length of simulated rail with the original model, whereas this value is much more constant (0.15\%) with the MRM which agrees better with real straight track corrugation.

\subsection{Local results obtained with both OM and MRM}

Thus the influence of MRM on the global results and how it acts on the contact nodes has been demonstrated. In this part, three nodes in different contact positions (Fig. 6) have been chosen to study the contact statements.

First, with the original model, the local frictional power in the contact was calculated (Fig. 7). The results show that the nodes at the entrance and the exit of the contact have very low frictional power; the values vary within a range of $400-600 \mathrm{~W}$ at a fixed frequency of $527 \mathrm{~Hz}$ (Figs. 7a and 7c). On the contrary, the node at the center of the contact is affected by a higher frictional power whose amplitude reaches about $1100 \mathrm{~W}$ (Fig. 7b) but with a tendency to decay within $0.01 \mathrm{~s}$ of the simulation. More precisely, the amplitude decreased from 

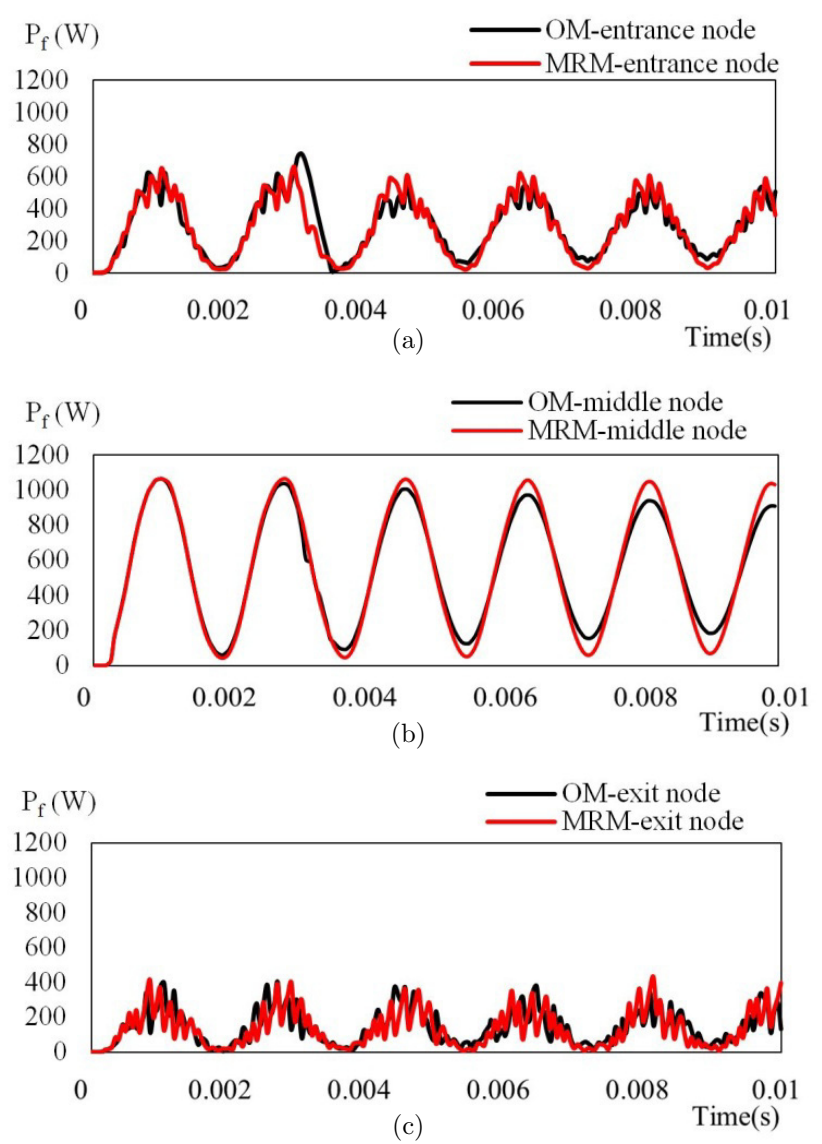

Fig. 6. Nodes at the wheel-rail contact.
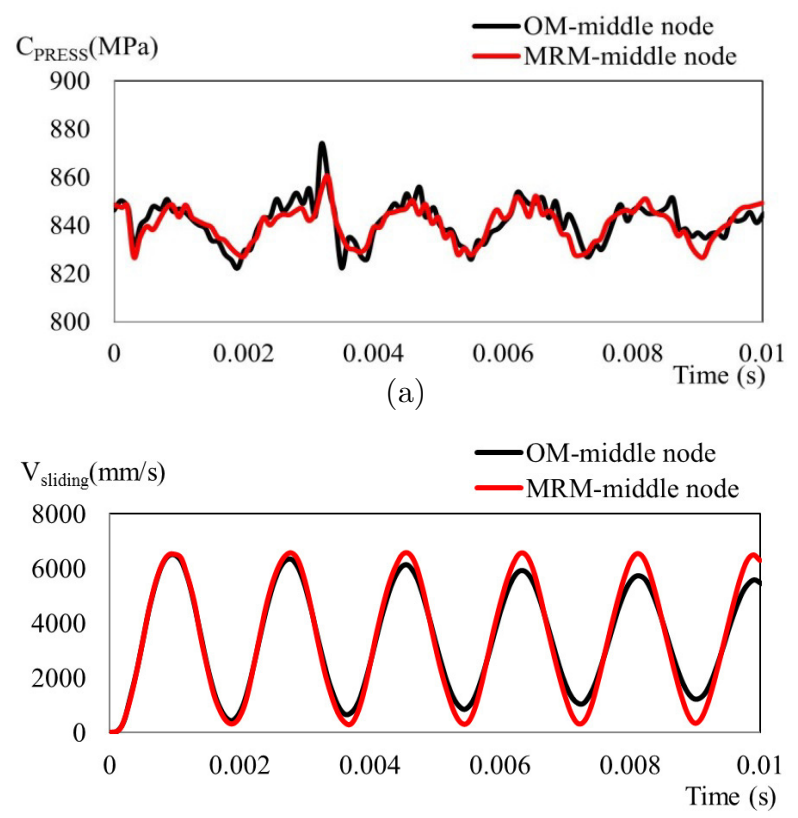

(b)

Fig. 7. Comparison of the local frictional power between the original model $(\mathrm{OM})$ and the mass redistribution model (MRM) at (a) the entrance node, (b) the center node and (c) the exit node of the contact.

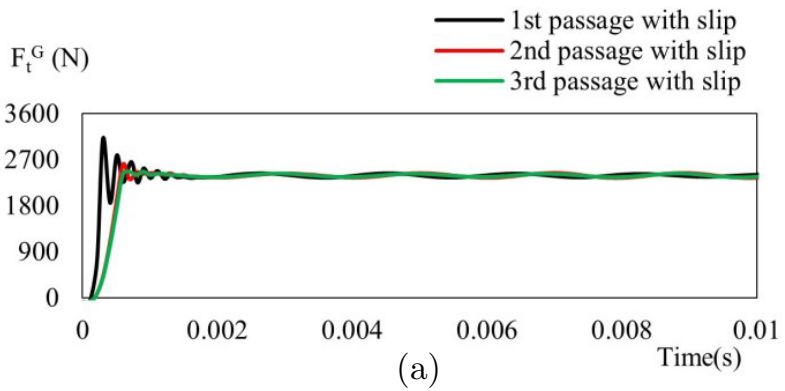

$\mathrm{P}_{\mathrm{f}}^{\mathrm{G}}(\mathrm{W})$

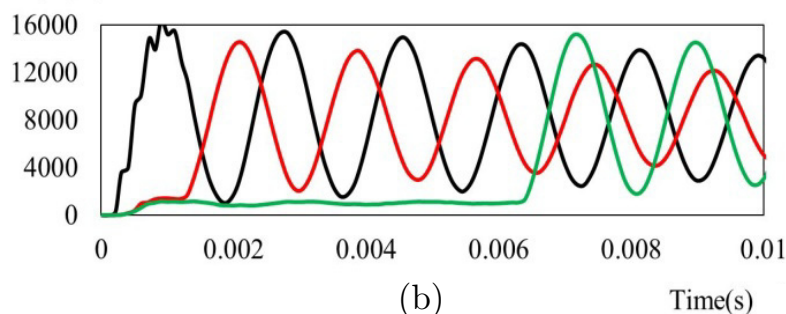

Fig. 8. Comparison of the (a) local contact pressure and (b) sliding velocity at the center node between the original model $(\mathrm{OM})$ and the mass redistribution model (MRM).

$1100 \mathrm{~W}$ to $900 \mathrm{~W}$ (Fig. 7b). The 3 sub-figures (Fig. 7) show that the frictional power is mainly localized at the center of the contact. The energy dissipated by the nodes at the center of the contact is higher than elsewhere in the contact.

Secondly, the results presented in Figures $7 \mathrm{a}$ and 7c show a high frequency around $5000 \mathrm{~Hz}$, caused by the instability of contact status whereas the entrance node and exit node shift instantaneously between stick status and slip status. Moreover, with the mass redistribution method, the value of the frictional power at both the entrance and exit of the contact is hardly affected (Figs. 7a and 7c). The space discretization problem is not clear at these nodes since the sliding velocity approaches zero at both the entrance node and at the exit node. Since neither the entry node nor the exit node is affected, it is the frictional power at the center node that is influenced. The decay of the amplitude seems to be minimized, and the peak value of each period is kept at about $1100 \mathrm{~W}-1050 \mathrm{~W}$. At the center node, the contact pressure and especially the sliding velocity (Figs. 8a and 8b) were also considerably influenced by the mass redistribution method.

Thus, from observing the local contact nodes, it was seen that the MRM had a significant effect on the contact spatial discretization problem, especially in the contact center.

\section{Results obtained with multiple wheel passes for both OM and MRM}

The results of the previous study [19] showed the onset of the periodical defect on the rail surface with OM for single wheel passes. Thus, by using both OM and MRM with multiple wheel passes, we expect to find 

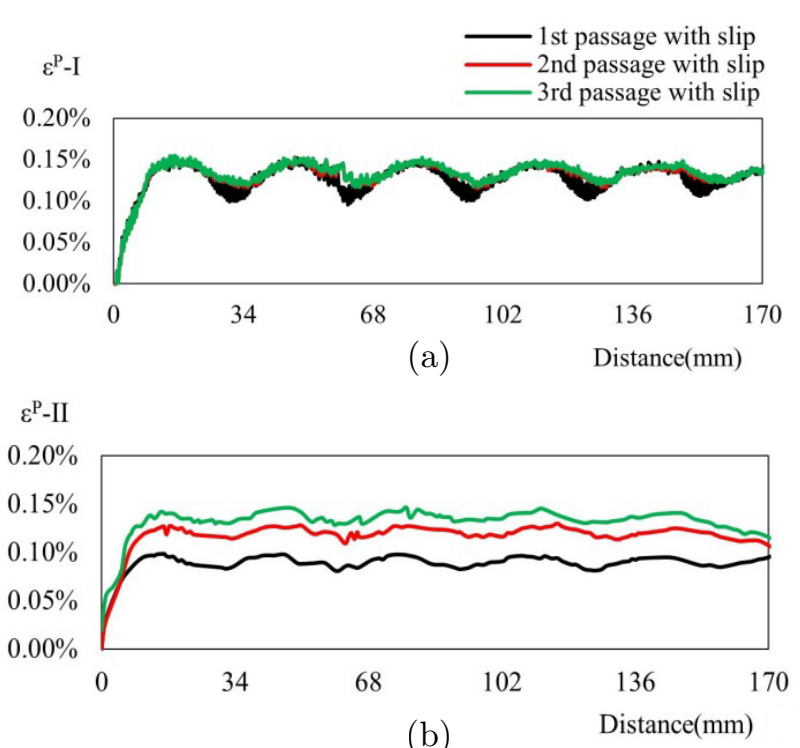

Fig. 9. Evolution of (a) the global tangential contact force $F_{t}^{G}$ and (b) the global frictional power $P_{f}^{G}$ with three wheels passes in OM.

an improvement in reproducing both the onset and evolution of the corrugation defect realistically. The previous 2D finite element model with MRM was extended to allow two additional wheel passes. Each additional passage used the same simulation protocol (see Fig. 1), as presented in Section 2.1. Before the transient condition was applied, the wheel-rail contact was in rolling without sliding conditions and subjected to constant loading. Thus the transient condition was applied to reach rolling with sliding conditions in the contact with the same time for each wheel. This simulation protocol appeared to be the severest for increasing the corrugation amplitude. The simulation parameters are summarized in Table 2. The same elasto-plastic law was used for the rail material: $\sigma_{\text {ep }}=455+250 \varepsilon^{\mathrm{P}}$.

\subsection{Additional wheel passages with slip in the original model (OM)}

The simulation was performed for the 2 nd and $3 \mathrm{rd}$ wheel passage with the slip ratio imposed. The amplitude of the global tangential contact force $F_{t}^{G}$ (see Fig. 9a) did not change during the slip instability state after the first millisecond of simulation. For the following wheel passage, the amplitude of $F_{t}^{G}$ was not as high as in the previous passage. The slip phase in each passage remained at the same frequency as the previous passage, as highlighted by Saulot et al. [19] (See Sect. 2.3).

The amplitude of $P_{f}^{G}$ seemed to slightly decrease after the passage of both the second and third wheels. This evolution might have been due to the accommodation of the slip velocity at the interface which was slightly modified due to the periodical geometry formed after the passage of the first wheel.
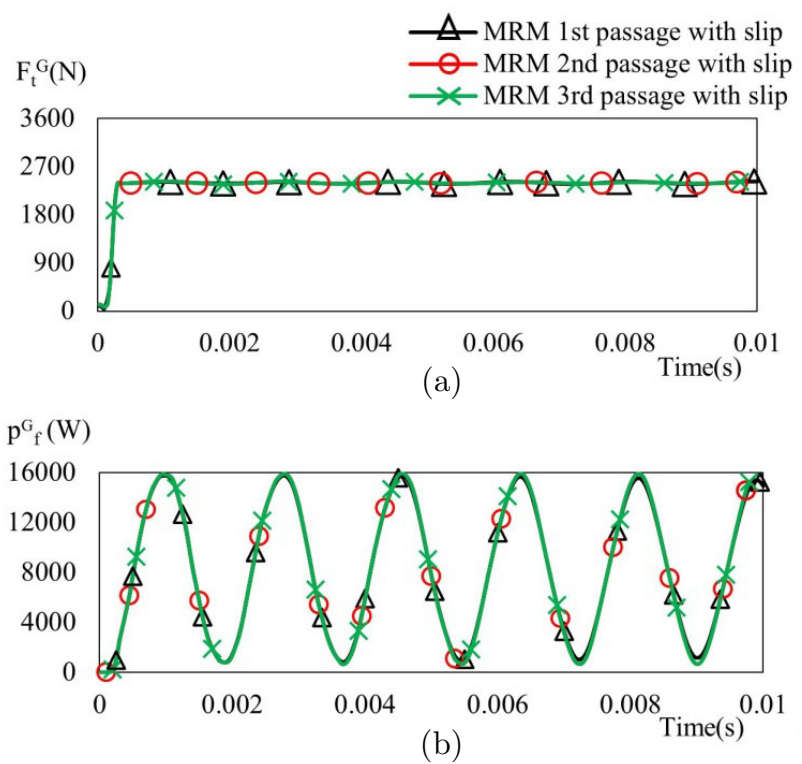

Fig. 10. Plastic deformation of rail: (a) on the rail surface and (b) in depth for three wheels passing in OM.

There was no major change in the amplitude of the plastic deformation $\varepsilon^{\mathrm{P}}$-I on the rail surface (see Fig. 10a) linked to the passage of multiple wheels. The value of the plastic deformation varies in the same range from $0.10 \%$ to $0.15 \%$, and there was a $0.01 \%$ difference in the peak value of plastic deformation from one passage to another. The passage of multiple wheels with slip had very slight influence on rail surface plastic deformation.

On the contrary, the amplitude of $\varepsilon^{\mathrm{P}}$-II (see Fig. 10b) in terms of rail depth increased with the number of wheel passes, with the occurrence of slip instability. Consequently, the relative amplitude $\Delta \varepsilon^{\mathrm{P}}$-II between the crest and trough of the corrugation defect, see Figure 10b, also increased with the number of wheel passes. Indeed, this value, measured in Figure 10b, rose from the first wheel pass to the third wheel pass. This indicates that the periodical plastic deformation inside the rail was increasingly apparent with the repetition of such transient conditions.

From the qualitative viewpoint, these results show good agreement with on-site observations of real corrugation defects on rail surfaces [10]. The plastic deformation obtained shows the same tendency as the trough and crest defect observed along the rail surface. Indeed, this defect increases with both the number of wheel passes and the repetition of contact conditions. The rails affected by straight track corrugation mostly belong to urban networks characterized by similar train types and traffic conditions (stops, crossings, etc.) that imply reproducible global contact conditions for each train.

\subsection{Additional wheel passages with slip in the Mass Redistribution Model (MRM)}

Using the same simulation protocol, multiple wheels passes under slip instability state were reproduced with 


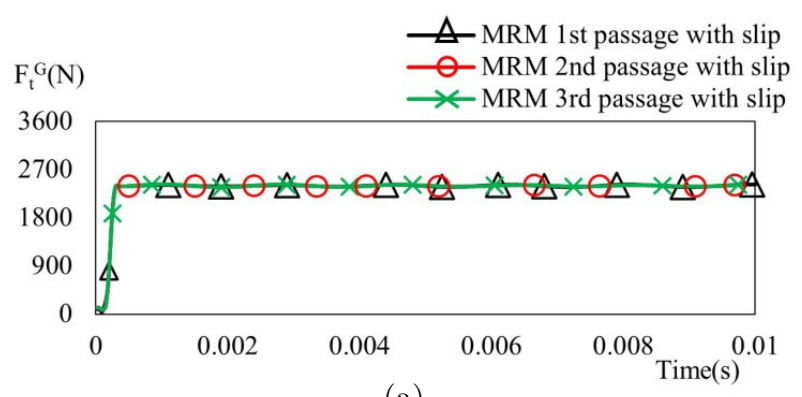

(a)

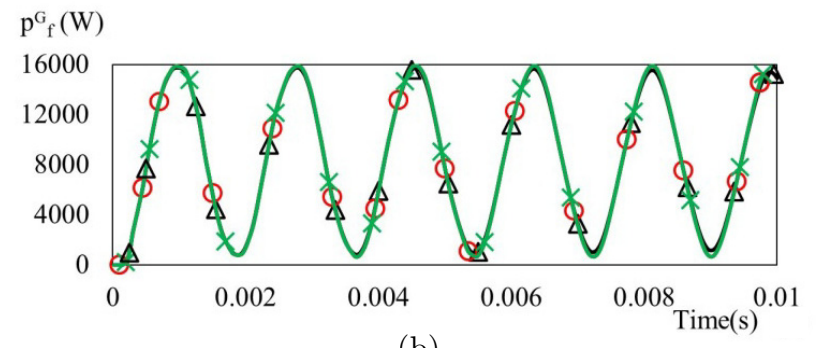

(b)

Fig. 11. Evolution of (a) global tangential contact force $F_{t}^{G}$ and (b) global frictional power $P_{f}^{G}$ of three wheels passing in MRM.

MRM. Both the global tangential contact force and frictional power were investigated. There was no significant change in the amplitude of the global tangential contact force $F_{t}^{G}$ (see Fig. 11a) in slip oscillatory state compared with the results obtained with the original model (lower than 5\%). On the contrary, unlike the decreased amplitude and dissipative energy in the original model (OM), the vibration trend and peak value of $P_{f}^{G}$ remained constant at $16000 \mathrm{~W}$ in MRM. Thus the results with OM (Fig. 9b) were due to the discretization problem in displacement, and not to the accommodation of slip velocity. The application of MRM improved the solution to this problem and its results did not show the energy disturbance for the first three wheel passes. There was no marked change in the amplitude of the plastic deformation on the rail surface (see Fig. 12a) for the three wheels passes. The value of the plastic deformation $\varepsilon^{\mathrm{P}}-\mathrm{I}$ varied in the same range from $0.10 \%$ to $0.15 \%$ on the rail surface, and the peak value of plastic deformation on each wavelength stayed at $0.15 \%$ and did not decay after three passes in comparison with the results obtained in original model (OM).

Regarding rail depth (see Fig. 12b), the amplitude of $\varepsilon^{\mathrm{P}}$-II was higher than the previous passage in slip oscillatory state (about $20 \%$ between two passages). These curves (see Fig. 12b) agree with the results obtained with the original model (see Fig. 10b). The different visualizations of the results of $\varepsilon^{\mathrm{P}}$-I and $\varepsilon^{\mathrm{P}}$-II with MRM indicate that the mass redistribution method had a significant influence on the inertia effect in the contact area. Thus the results of $\varepsilon^{\mathrm{P}}$-I differed between OM and MRM, and there was little difference in the results of $\varepsilon^{\mathrm{P}}$-II between $\mathrm{OM}$ and MRM. The results obtained with MRM showed that the inertia effect at the contact area was lower. Therefore
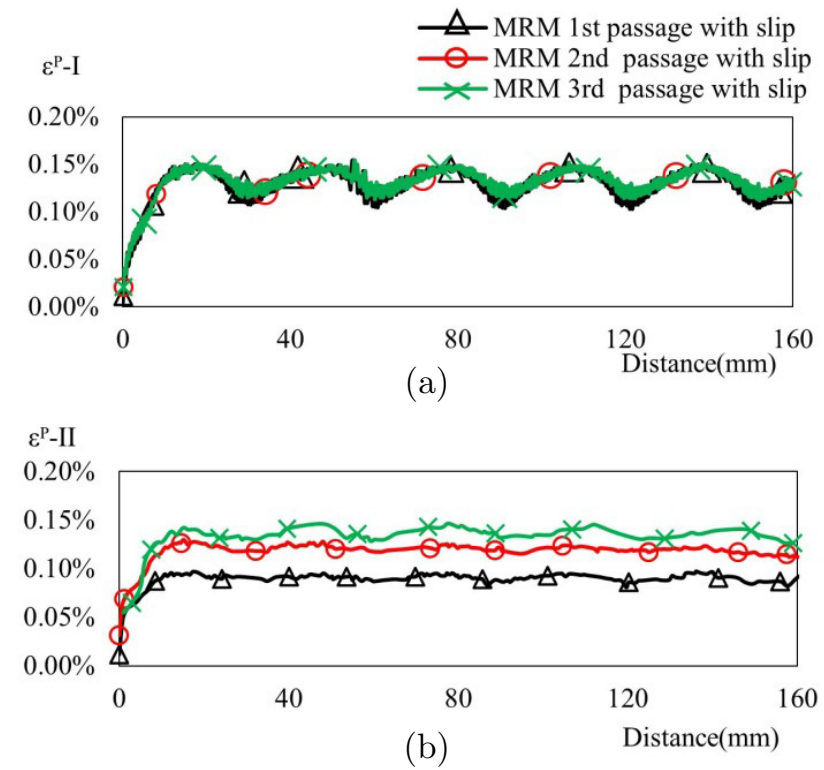

Fig. 12. Plastic deformation of a rail: (a) on the rail surface and (b) inside the rail with three wheels passing in MRM.

the model with MRM reproduced the corrugation defects more realistically.

\section{Conclusion}

An improved 2D finite element model of wheel-rail contact was developed using the Mass Redistribution Method (MRM). This method not only solved the numerical dissipation of energy in the contact model but also ensured that it converged. The improved model was used to reproduce a periodical rail defect known as straight track corrugation. The results obtained with MRM were more realistic than those obtained with the previous model used by Saulot et al. [19].

Both the origin and onset of this defect was investigated under both multiple wheels passages and transient conditions. The origin of corrugation was linked to the wheel-rail contact instability state that occurs under such transient conditions and more precisely during the slip instability state. For several wheel passages, although the results were obtained for only the first three wheel passages under transient conditions, the information obtained in the model showed the trend of corrugation development. Furthermore, the use of MRM situated the consequences of each wheel in phase, thereby providing a better demonstration of the trend towards the corrugation defect than that obtained with the original model (OM) developed by Saulot et al. [19].

Future work is planned to continue improving this finite element model of wheel-rail contact using MRM. The first improvement will focus on 3D modeling to approach the reality of the wheel-rail even more closely. The second improvement will focus on the use of a coupled EulerianLagrangian method to optimize computation time. 
Acknowledgements. The authors would like to thank the Chinese Scholarship Council for funding this research. The authors would also like to thank Pr. Y. Renard from LaMCoSICJ of INSA-Lyon for his helpful comments and discussions. The authors would finally like to thank Dr. A. Duval from LaMCoS of INSA-Lyon for his technical support with the use of ABAQUS. This paper has been proofread and corrected by a native English speaker.

\section{References}

[1] Y. Sato, A. Matsumoto, K. Knothe, Review on rail corrugation studies, Wear 253 (2002) 130-139

[2] Q.Y. Liu, B. Zhang, Z.R. Zhou, An experimental study of rail corrugation, Wear 255 (2003) 1121-1126

[3] T. Mazilu, Green's functions for analysis of dynamic response of wheel/rail to vertical excitation, J. Sound Vib. 306 (2007) 31-58

[4] Z. Yan, A. Gu, W. Liu, V.L. Markine, Q. Liang, Effects of wheelset vibration on initiation and evolution of rail short-pitch corrugation, J. Cent. South Univ. 19 (2012) 2681-2688

[5] F. Alwahdi, F.J. Franklin, A. Kapoor, The effect of partial slip on the wear rate of rails, Wear 258 (2005) 10311037

[6] A. Böhmer, T. Klimpel, Plastic deformation of corrugated rails - a numerical approach using material data of rail steel, Wear 253 (2002) 150-161

[7] A. Ekberg, E. Kabo, Fatigue of railway wheels and rails under rolling contact and thermal loading - an overview, Wear 258 (2005) 1288-1300

[8] D.F. Cannon, H. Pradier, Rail rolling contact fatigue Research by the European Rail Research Institute, Wear 191 (1996) 1-13

[9] A. Saulot, S. Descartes, Y. Berthier, Sharp curved track corrugation: From corrugation observed on-site, to corrugation reproduced on simulators, Tribol. Int. 42 (2009) 1691-1705

[10] E. Wild, L. Wang, B. Hasse, T. Wroblewski, G. Goerigk, A. Pyzalla, Microstructure alterations at the surface of a heavily corrugated rail with strong ripple formation, Wear 254 (2003) 876-883

[11] M. Hiensch, J.C.O. Nielsen, E. Verheijen, Rail corrugation in The Netherlands-measurements and simulations, Wear 253 (2002) 140-149

[12] W. Cai, Z. Wen, X. Jin, W. Zhai, Dynamic stress analysis of rail joint with height difference defect using finite element method, Eng. Fail. Anal. 14 (2007) 1488-1499
[13] X. Jin, Z. Wen, K. Wang, W. Zhang, Effect of a scratch on curved rail on initiation and evolution of rail corrugation, Tribol. Int. 37 (2004) 385-394

[14] X. Jin, Z. Wen, W. Zhang, Z. Shen, Numerical simulation of rail corrugation on a curved track, Comput. Struct. 83 (2005) 2052-2065

[15] W. Li, G. Xiao, Z. Wen, X. Xiao, X. Jin, Plastic deformation of curved rail at rail weld caused by train-track dynamic interaction, Wear 271 (2011) 311-318

[16] X. Jin, X. Xiao, Z. Wen, J. Guo, M. Zhu, An investigation into the effect of train curving on wear and contact stresses of wheel and rail, Tribol. Int. 42 (2009) 475-490

[17] X.S. Jin, Z.F. Wen, K.Y. Wang, Z.R. Zhou, Q.Y. Liu, C.H. Li, Three-dimensional train-track model for study of rail corrugation, J. Sound Vib. 293 (2006) 830-855

[18] C. Andersson, A. Johansson, Prediction of rail corrugation generated by three-dimensional wheel-rail interaction, Wear 257 (2004) 423-434

[19] A. Saulot, L. Baillet, Dynamic Finite Element Simulations for Understanding Wheel-Rail Contact Oscillatory States Occurring Under Sliding Conditions, J. Tribol. 128 (2006) 761

[20] M. Ertz, K. Knothe, A comparison of analytical and numerical methods for the calculation of temperatures in wheel / rail contact, Wear 253 (2002) 498-508

[21] Y.-C. Chen, S.-Y. Lee, Elastic-Plastic Wheel-Rail Thermal Contact on Corrugated Rails During Wheel Braking, J. Tribol. 131 (2009) 011401

[22] H.B. Khenous, P. Laborde, Y. Renard, Mass redistribution method for finite element contact problems in elastodynamics, Eur. J. Mech. - A/Solids 27 (2008) 918-932

[23] D. Doyen, A. Ern, Convergence of a space semi-discrete modified mass method for the dynamic Signorini problem, Commun. Math. Sci. 7 (2009) 1063-1072

[24] T. Ligursky, Y. Renard, A Well-Posed SemiDiscretization of Elastodynamic Contact Problems with Friction, Q.J. Mech. Appl. Math. 64 (2011) 215-238

[25] Y. Renard, The singular dynamic method for constrained second order hyperbolic equations: Application to dynamic contact problems, J. Comput. Appl. Math. 234 (2010) 906-923

[26] JCO. Nielsen, R. Lunden, A. Johnansson, T. Vernersson, Train-track interaction and mechanisms of irregular wear on wheel and rail surfaces, Veh. Syst. Dyn. 40 (2003) 3-54

[27] A. Matsumoto, Y. Sato, H. Ono, M. Tanimoto, Y. Oka, E. Miyauchi, Formation mechanism and countermeasures of rail corrugation on curved track, Wear 253 (2002) 178184 